\title{
Radiation of a charge flying in a partially loaded dielectric section of a waveguide
}

\author{
Aleksandra A. Grigoreva, ${ }^{1}$ Andrey V. Tyukhtin, ${ }^{1}$ Viktor V. Vorobev, ${ }^{1}$ \\ Sergey N. Galyamin, ${ }^{1}$ and Sergey Antipov ${ }^{2}$ \\ ${ }^{1}$ Saint Petersburg State University, 7/9 Universitetskaya nab., St. Petersburg 199034, Russia \\ ${ }^{2}$ Euclid Techlabs, 365 Remington blvd, Bolingbrook, Illinois 60440, USA \\ and Argonne National Laboratory, 9700 S. Cass ave, Lemont, Illinois 60439, USA
}

(Received 22 November 2017; published 12 March 2018)

\begin{abstract}
We consider the electromagnetic field of a point charged particle moving along the axis of a cylindrical waveguide from a homogeneously filled area to a dielectric loading area having an axially symmetrical channel. We are interested in studying the Cherenkov radiation excited in the bilayer area. The solution is performed by expanding the field in each area in a series of orthogonal eigenmodes. The main attention is focused on investigation of the wave field in the bilayer section. We show that, at a given observation point, the "reduced wakefield" is simplified with time (the number of modes decreases). The obtained results are generalized for the case of a bunch with Gaussian longitudinal profile. The typical numerical results for wakefield formation process are presented. These results agree with simulations done by the industry standard electromagnetic code CST Particle Studio.
\end{abstract}

DOI: 10.1103/PhysRevAccelBeams.21.031302

\section{INTRODUCTION}

Considerable attention is currently focused on the development of new methods of charged particle acceleration, in particular, the wakefield acceleration technique in dielectric loaded waveguides. This technique is based on the use of a Cherenkov radiation generated by the charged particle bunch moving through the waveguide $[1,2]$. In this connection, the possibility to use rectangular [3-5] and cylindrical [6,7] waveguides containing a vacuum channel as an accelerating structure is considered. Analysis of the wakefield formation process is significant for this acceleration method.

Note that the investigation of electromagnetic fields produced by charged particles in a dielectric-loaded waveguide with channel is of interest for development of methods of bunch diagnostics as well. For example, the bunch length measurement method by use of bilayer circular waveguides was presented in [8]. Reference [9] is devoted to the method for determination of particle energy. The proposed method is based on the dependence of the mode frequency of a cylindrical regular bilayer waveguide on Lorentz factor.

The electromagnetic fields generated by moving charges in cylindrical waveguides with transverse boundaries were

Published by the American Physical Society under the terms of the Creative Commons Attribution 4.0 International license. Further distribution of this work must maintain attribution to the author(s) and the published article's title, journal citation, and DOI. investigated earlier in the series of papers [10-12]. However, the presence of a vacuum channel was not considered in these works. This assumption is justified if the channel radius is much less than the waveguide one. However, if the channel radius is comparable with the waveguide one then the problem is complicated principally because two waveguide parts possess different sets of eigenmodes.

In the present paper we investigate the electromagnetic field of a point charge and Gaussian bunch moving with uniform velocity along the cylindrical waveguide axis. The waveguide consists of two semi-infinite parts: a homogeneously filled part and a dielectric part with coaxial channel and dielectric layer. The charge moves from the homogeneous section into the bilayer one.

It should be noted that a similar structure was considered earlier for the problem of axially symmetrical transversemagnetic mode launching on the transverse boundary in the waveguide [13]. In that work we described the mode transformation effect caused by the waveguide irregularity. We also studied the case of a charge moving from the bilayer area to the vacuum one [14]. The main attention was focused on the so-called Cherenkov-transition radiation effect when Cherenkov radiation excited in the bilayer area penetrates through the transverse boundary. In particular, we showed that it is possible to generate both monochromatic and multifrequency radiation in the vacuum waveguide area.

The present work is devoted to the case of the "opposite" charge motion which results in different physical effects. We assume that the charge velocity is more than the light velocity in the cylindrical layer but less than the light 
velocity in the channel and in the homogeneous part of the waveguide. Therefore, Cherenkov radiation is excited in the bilayer waveguide area, but it is not excited in the homogeneous one. Note that the wave field of the charge in the regular cylindrical waveguide with channel (the socalled "wakefield") is well studied [1,2]. Here we focus on analytical and numerical investigation of process of the wakefield formation in the bilayer waveguide area.

\section{ANALYTICAL TREATMENT}

We consider the infinite cylindrical waveguide of radius $a$ composed of the area with relative permittivity $\varepsilon_{1}$, relative permeability $\mu_{1}$, and bilayer area with dielectric filling $\left(\varepsilon_{d}, \mu_{d}\right)$ and coaxial channel $\left(\varepsilon_{c}, \mu_{c}\right)$ of radius $b$ (see Fig. 1). All media are isotropic, homogeneous, and nondispersive. The dissipation in the media is negligible. The external waveguide surface is perfectly conducting. The cylindrical coordinates are used. The point particle with the charge $q$ uniformly moves along the waveguide axis with velocity $\vec{v}=c \beta \vec{e}_{z}$ ( $c$ means the speed of light in vacuum) and intersects the boundary $z=0$ at the instant time $t=0$.

We consider the situation when $n_{d} \beta>1, n_{c} \beta<1, n_{1} \beta<$ $1\left(n_{i}=\sqrt{\varepsilon_{i} \mu_{i}}, i=c, d, 1\right)$, i.e., Cherenkov radiation is generated only in the area $z>0, r>b$. Initially we put for generality that $n_{1}$ and $n_{c}$ are different from 1 , but further we will study in more detail the case of the vacuum area $z<0$ and the vacuum channel since this situation is of main interest for practice.

The electromagnetic field in each waveguide area can be presented as a sum of two terms:

$$
\vec{E}_{1,2}=\vec{E}_{1,2}^{(q)}+\vec{E}_{1,2}^{(b)}, \quad \vec{H}_{1,2}=\vec{H}_{1,2}^{(q)}+\vec{H}_{1,2}^{(b)}
$$

The first summand with superscript $(q)$ is the field of the particle in the infinite regular waveguide. It is called a "forced" field by Ginzburg [15]. The second summand (b) ("free" field) is determined by the influence of the transverse boundary. The subscripts 1 or 2 denotes the area $z<0$ and $z>0$ correspondingly.

The forced fields in both areas have been investigated in the literature $[2,9,16]$. We give here expressions only for longitudinal field components:

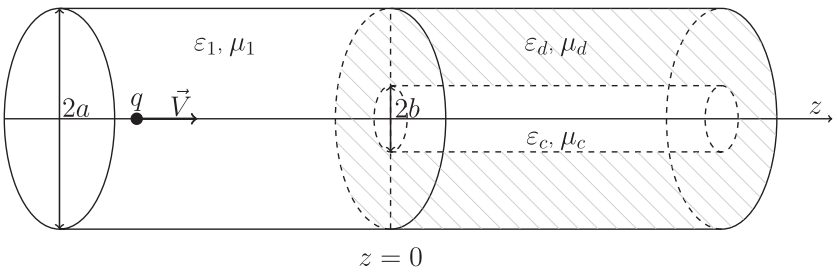

FIG. 1. Composite waveguide structure.

$$
\begin{aligned}
E_{1 z}^{(q)}= & -\frac{q}{2 v^{2} \varepsilon_{1}}\left(n_{1}^{2} \beta^{2}-1\right) \int_{-\infty}^{\infty} \omega \exp \left(i \frac{\omega}{v} \zeta\right) \\
& \times\left(H_{0}^{(1)}\left(s_{1} r\right)-\frac{H_{0}^{(1)}\left(s_{1} a\right)}{J_{0}\left(s_{1} a\right)} J_{0}\left(s_{1} r\right)\right) d \omega, \\
E_{2 z}^{(q)}= & \frac{i q}{\pi v^{2}}\left(n_{2}^{2} \beta^{2}-1\right) \int_{-\infty}^{+\infty} \omega \exp \left(i \frac{\omega}{v} \zeta\right) \Phi^{(q)}(r, \omega) d \omega,
\end{aligned}
$$

where

$$
\begin{aligned}
n_{2} & =\sqrt{\varepsilon_{2} \mu_{2}}, \quad \varepsilon_{2}, \mu_{2}=\left\{\begin{array}{l}
\varepsilon_{c}, \mu_{c} \quad r \leq b \\
\varepsilon_{d}, \mu_{d} r \geq b,
\end{array} \quad \zeta=z-v t,\right. \\
k(\omega) & =\frac{|\omega|}{v} \sqrt{1-n_{c}^{2} \beta^{2}}, \quad \operatorname{Re}(k)>0, \\
s(\omega) & =\frac{\omega}{v} \sqrt{n_{d}^{2} \beta^{2}-1}, \quad \operatorname{Im}(s) \geq 0, \\
s_{1}(\omega) & =\frac{\omega}{v} \sqrt{n_{1}^{2} \beta^{2}-1}, \quad \operatorname{Im}\left(s_{1}\right) \geq 0, \\
\Phi^{(q)}(r, \omega) & =\varepsilon_{c}^{-1}\left[K_{0}(k r)+\chi^{(q)} I_{0}(k r)\right] r \leq b, \\
\Phi^{(q)}(r, \omega) & =\frac{i \pi \nu(q)}{2 \varepsilon_{d}}\left(H_{0}^{(1)}(s r)-\frac{H_{0}^{(1)}(s a)}{J_{0}(s a)} J_{0}(s r)\right) r \geq b, \\
\psi_{0,1}(x) & =J_{1,0}(x b) Y_{0}(x a)-J_{0}(x a) Y_{1,0}(x b), \\
\chi^{(q)} & =\frac{\varepsilon_{c} s K_{1}(k b) \psi_{1}(s)+\varepsilon_{d} k K_{0}(k b) \psi_{0}(s)}{\varepsilon_{c} s I_{1}(k b) \psi_{1}(s)-\varepsilon_{d} k I_{0}(k b) \psi_{0}(s)}, \\
\nu^{(q)} & =-\frac{J_{0}(s a)}{\pi b s} \frac{J_{d} k I_{1}(k b) \psi_{1}(s)-\varepsilon_{d} k I_{0}(k b) \psi_{0}(s)}{\varepsilon_{c}} .
\end{aligned}
$$

Here $J_{0,1}(x), Y_{0,1}(x)$ are the Bessel and Neumann functions, respectively, $H_{0,1}^{(1)}(x)$ are Hankel functions of the first kind, $I_{0,1}(x), K_{0,1}(x)$ are modified Bessel and Hankel functions, respectively.

The free field components can be written as a series of eigenfunctions of the transverse operator. According to this method, a free field takes the form

$$
\begin{gathered}
\vec{E}_{1,2}^{(b)}=\sum_{n=1}^{\infty} \vec{E}_{1 n, 2 n}^{(b)}, \quad \vec{H}_{1,2}^{(b)}=\sum_{n=1}^{\infty} \vec{H}_{1 n, 2 n}^{(b)}, \\
\left\{\begin{array}{c}
H_{1 \varphi n}^{(b)} \\
E_{1 z n}^{(b)} \\
E_{1 r n}^{(b)}
\end{array}\right\}=\frac{\eta_{n}}{\mu_{1} a} \int_{-\infty}^{+\infty} B_{n}^{(1)}(\omega) \exp \left[i \Phi_{1 n}(\omega)\right] \\
\times\left\{\begin{array}{c}
\frac{i c \eta_{n}}{\varepsilon_{1} a \omega} J_{0}\left(\eta_{n} \frac{r}{a}\right) \\
-\frac{c h_{n}^{(1)}(\omega)}{\varepsilon_{1} \omega} J_{1}\left(\eta_{n} \frac{r}{a}\right)
\end{array}\right\} d \omega,
\end{gathered}
$$




$$
\begin{aligned}
\left\{\begin{array}{c}
H_{2 \varphi n}^{(b)} \\
E_{2 z n}^{(b)} \\
E_{2 r n}^{(b)}
\end{array}\right\}= & \int_{-\infty}^{+\infty} B_{n}^{(2)}(\omega) \exp \left[i \Phi_{2 n}(\omega)\right] \\
& \times\left\{\begin{array}{c}
F_{n}(r, \omega) \\
\frac{i c}{\varepsilon_{2} \omega}\left(\frac{F_{n}(r, \omega)}{r}+\frac{\partial F_{n}(r, \omega)}{\partial r}\right) \\
\frac{c h_{n}^{(2)}(\omega)}{\varepsilon_{2} \omega} F_{n}(r, \omega)
\end{array}\right\} d \omega .
\end{aligned}
$$

Here $\Phi_{1 n, 2 n}=-\omega t \mp h_{n}^{(1,2)}(\omega) z$, functions $B_{n}^{(1,2)}(\omega)$ are unknown mode excitation coefficients, $\eta_{n}$ is the zero of Bessel function $J_{0}\left(\eta_{n}\right)=0$;

$$
\begin{aligned}
h_{n}^{(1)} & =\sqrt{\frac{\omega^{2} n_{1}^{2}}{c^{2}}-\frac{\eta_{n}^{2}}{a^{2}}}, \quad \operatorname{Im}\left(h_{n}^{(1)}\right) \geq 0 ; \\
h_{n}^{(2)}= & \sqrt{\frac{\omega^{2} n_{c, d}^{2}}{c^{2}}-\alpha_{c n, d n}^{2}, \quad \operatorname{Im}\left(h_{n}^{(2)}\right) \geq 0 ;} \\
F_{n}(r, \omega)= & J_{1}\left(\alpha_{c n} r\right) r \leq b, \\
F_{n}(r, \omega)= & \frac{i J_{0}\left(\alpha_{d n} a\right) J_{1}\left(\alpha_{c n} b\right)}{\psi_{0}\left(\alpha_{d n}\right)}\left(H_{1}^{(1)}\left(\alpha_{d n} r\right)\right. \\
& \left.-\frac{H_{0}^{(1)}\left(\alpha_{d n} a\right)}{J_{0}\left(\alpha_{d n} a\right)} J_{1}\left(\alpha_{d n} r\right)\right) r \geq b .
\end{aligned}
$$

Note that functions $F_{n}(r, \omega)$ possess the orthogonality property (bar means complex conjunction):

$$
\int_{0}^{a} \bar{F}_{n}(r, \omega) F_{m}(r, \omega) \frac{r}{\varepsilon_{2}} d r=\delta_{n m} G_{n}(\omega),
$$

where

$$
\begin{aligned}
G_{n}(\omega)= & \frac{b^{2}}{2 \varepsilon_{c}}\left[J_{1}^{2}\left(\alpha_{c n} b\right)+J_{0}^{2}\left(\alpha_{c n} b\right)-\frac{2}{\alpha_{c n} b} J_{0}\left(\alpha_{c n} b\right) J_{1}\left(\alpha_{c n} b\right)\right] \\
& +\frac{J_{1}^{2}\left(\alpha_{c n} b\right)}{2 \varepsilon_{d} \psi_{0}^{2}\left(\alpha_{d n}\right)}\left[\frac{4}{\pi^{2} \alpha_{d n}^{2}}+\frac{2 b}{\alpha_{d n}} \psi_{0}\left(\alpha_{d n}\right) \psi_{1}\left(\alpha_{d n}\right)-b^{2} \psi_{0}^{2}\left(\alpha_{d n}\right)-b^{2} \psi_{1}^{2}\left(\alpha_{d n}\right)\right] \text { for } \alpha_{c n} \in \mathbb{R}, \\
G_{n}(\omega)= & \frac{b^{2}}{2 \varepsilon_{c}}\left[I_{1}^{2}\left(\left|\alpha_{c n}\right| b\right)-I_{0}^{2}\left(\left|\alpha_{c n}\right| b\right)+\frac{2}{\left|\alpha_{c n}\right| b} I_{0}\left(\left|\alpha_{c n}\right| b\right) \times I_{1}\left(\left|\alpha_{c n}\right| b\right)\right] \\
+ & \frac{I_{1}^{2}\left(\left|\alpha_{c n}\right| b\right)}{2 \varepsilon_{d} \psi_{0}^{2}\left(\alpha_{d n}\right)}\left[\frac{4}{\pi^{2} \alpha_{d n}^{2}}+\frac{2 b}{\alpha_{d n}} \psi_{0}\left(\alpha_{d n}\right) \psi_{1}\left(\alpha_{d n}\right)-b^{2} \psi_{0}^{2}\left(\alpha_{d n}\right)-b^{2} \psi_{1}^{2}\left(\alpha_{d n}\right)\right] \text { for } \alpha_{c n}=i\left|\alpha_{c n}\right| .
\end{aligned}
$$

Coefficients $\alpha_{c n, d n}$ can be determined as follows. The requirement $E_{2 z}^{(b)}=0$ at the surface $r=a$ and the continuity conditions for $E_{2 z}^{(b)}$ and $H_{2 \varphi}^{(b)}$ at the surface $r=b$ result in the relation $\alpha_{c n}^{2}=\omega^{2}\left(n_{c}^{2}-n_{d}^{2}\right) / c^{2}+\alpha_{d n}^{2}$ and the following dispersion equation, which can be regarded as an equation for coefficients $\alpha_{d n}$ :

$\varepsilon_{c} \alpha_{d n} J_{1}\left(\alpha_{c n} b\right) \psi_{1}\left(\alpha_{d n}\right)-\varepsilon_{d} \alpha_{c n} J_{0}\left(\alpha_{c n} b\right) \psi_{0}\left(\alpha_{d n}\right)=0$.

The mode excitation coefficients $B_{n}^{(1,2)}(\omega)$ can be found by using the continuity conditions for the tangential components of the total electromagnetic field [defined by the expression (1)] at the transverse boundary:

$$
\left.E_{1 r}\right|_{z=-0}=\left.E_{2 r}\right|_{z=+0},\left.\quad H_{1 \varphi}\right|_{z=-0}=\left.H_{2 \varphi}\right|_{z=+0} .
$$

Our main interest is to analyze the field generated in the bilayer area. For this purpose we multiply equalities (11) by the function $r J_{1}\left(\eta_{m} r / a\right)(m=1,2 \ldots)$, and integrate over the waveguide radius using the orthogonality property of eigenmodes in the region $z<0$ :

$$
\int_{0}^{a} J_{1}\left(\eta_{n} \frac{r}{a}\right) J_{1}\left(\eta_{m} \frac{r}{a}\right) r d r=\frac{a^{2} J_{1}^{2}\left(\eta_{m}\right)}{2} \delta_{n m} .
$$

In that way the following infinite systems can be obtained for coefficients $B_{n}^{(2)}(\omega)$ :

$$
\sum_{n=1}^{\infty} A_{m n} B_{n}^{(2)}=U_{m}, \quad m=1,2 \ldots
$$

$$
\begin{aligned}
A_{m n}= & \frac{a b}{\left(\alpha_{c n}^{2} a^{2}-\eta_{m}^{2}\right)\left(\alpha_{d n}^{2} a^{2}-\eta_{m}^{2}\right)}\left[\eta_{m} J_{0}\left(\eta_{m} \frac{b}{a}\right)\right. \\
& \left.\times J_{1}\left(\alpha_{c n} b\right) Q_{1}-a \alpha_{c n} J_{0}\left(\alpha_{c n} b\right) J_{1}\left(\eta_{m} \frac{b}{a}\right) Q_{2}\right],
\end{aligned}
$$




$$
\begin{aligned}
U_{m}= & -\frac{q \omega a \eta_{m}}{\pi v c\left(s_{1}^{2} a^{2}-\eta_{m}^{2}\right)}\left(1+h_{m}^{(1)} \frac{v}{\omega}\right)-\frac{q \omega a \varepsilon_{1} \eta_{m}}{\pi v c \varepsilon_{c}} \frac{1}{k^{2} a^{2}+\eta_{m}^{2}}\left(1+\frac{\varepsilon_{c}}{\varepsilon_{1}} h_{m}^{(1)} \frac{v}{\omega}\right) \\
& +\frac{q \omega a \varepsilon_{1} k}{\pi v c} \frac{1}{\left(k^{2} a^{2}+\eta_{m}^{2}\right)\left(s^{2} a^{2}-\eta_{m}^{2}\right)} \frac{s a J_{1}\left(\eta_{m} b / a\right) \psi_{1}(s) Q_{3}-\eta_{m} J_{0}\left(\eta_{m} b / a\right) \psi_{0}(s) Q_{4}}{\varepsilon_{c} s I_{1}(k b) \psi_{1}(s)-\varepsilon_{d} k I_{0}(k b) \psi_{0}(s)} .
\end{aligned}
$$

Coefficients $B_{n}^{(1)}(\omega)$ are expressed through $B_{n}^{(2)}(\omega)$ by the following way:

$$
\begin{aligned}
& B_{m}^{(1)}=\frac{2 \mu_{1}}{\eta_{m} a J_{1}^{2}\left(\eta_{m}\right)}\left\{\sum_{n=1}^{\infty} \frac{B_{n}^{(2)} a b}{\left(\alpha_{c n}^{2} a^{2}-\eta_{m}^{2}\right)\left(\alpha_{d n}^{2} a^{2}-\eta_{m}^{2}\right)}\left[\eta_{m} J_{0}\left(\eta_{m} \frac{b}{a}\right) J_{1}\left(\alpha_{c n} b\right) Q_{5}-a \alpha_{c n} J_{1}\left(\eta_{m} \frac{b}{a}\right) J_{0}\left(\alpha_{c n} b\right) Q_{6}\right]\right. \\
& +\frac{q \omega^{2} \eta_{m} a^{3}\left(n_{1}^{2}-n_{c}^{2}\right)}{\pi c^{3}\left(s_{1}^{2} a^{2}-\eta_{m}^{2}\right)\left(k^{2} a^{2}+\eta_{m}^{2}\right)} \\
& \left.-\frac{q k a}{\pi c F_{c h}(\omega)\left(s^{2} a^{2}-\eta_{m}^{2}\right)\left(k^{2} a^{2}+\eta_{m}^{2}\right)}\left[\varepsilon_{c} a s \psi_{1}(s) J_{1}\left(\eta_{m} \frac{b}{a}\right) Q_{7}-\varepsilon_{d} \eta_{m} \psi_{0}(s) J_{0}\left(\eta_{m} \frac{b}{a}\right) Q_{5}\right]\right\} \text {, } \\
& F_{c h}(\omega) \equiv \varepsilon_{c} s I_{1}(k b) \psi_{1}(s)-\varepsilon_{d} k I_{0}(k b) \psi_{0}(s), \\
& Q_{1}=\alpha_{d n}^{2} a^{2}\left(h_{m}^{(1)}+\frac{\varepsilon_{1}}{\varepsilon_{c}} h_{n}^{(2)}\right)-\alpha_{c n}^{2} a^{2}\left(h_{m}^{(1)}+\frac{\varepsilon_{1}}{\varepsilon_{d}} h_{n}^{(2)}\right)-\eta_{m}^{2} h_{n}^{(2)} \frac{\varepsilon_{1}}{\varepsilon_{c}}\left(1-\frac{\varepsilon_{c}}{\varepsilon_{d}}\right), \\
& Q_{2}=\alpha_{d n}^{2} a^{2}\left(h_{m}^{(1)}+\frac{\varepsilon_{1}}{\varepsilon_{c}} h_{n}^{(2)}\right)-\alpha_{c n}^{2} a^{2} \frac{\varepsilon_{d}}{\varepsilon_{c}}\left(h_{m}^{(1)}+\frac{\varepsilon_{1}}{\varepsilon_{d}} h_{n}^{(2)}\right)-\eta_{m}^{2} h_{m}^{(1)}\left(1-\frac{\varepsilon_{d}}{\varepsilon_{c}}\right), \\
& Q_{3}=k^{2} a^{2}\left(1+\frac{\varepsilon_{d}}{\varepsilon_{1}} h_{m}^{(1)} \frac{v}{\omega}\right)+s^{2} a^{2}\left(1+\frac{\varepsilon_{c}}{\varepsilon_{1}} h_{m}^{(1)} \frac{v}{\omega}\right)+\eta_{m}^{2} \frac{\varepsilon_{d}}{\varepsilon_{1}} h_{m}^{(1)} \frac{v}{\omega}\left(1-\frac{\varepsilon_{c}}{\varepsilon_{d}}\right), \\
& Q_{4}=k^{2} a^{2}\left(1+\frac{\varepsilon_{d}}{\varepsilon_{1}} h_{m}^{(1)} \frac{v}{\omega}\right)+\frac{\varepsilon_{d}}{\varepsilon_{c}} s^{2} a^{2}\left(1+\frac{\varepsilon_{c}}{\varepsilon_{1}} h_{m}^{(1)} \frac{v}{\omega}\right)+\eta_{m}^{2}\left(1-\frac{\varepsilon_{d}}{\varepsilon_{c}}\right), \\
& Q_{5}=\frac{\omega^{2} a^{2}}{c^{2}}\left(n_{d}^{2}-n_{c}^{2}\right) \\
& Q_{6}=a^{2}\left(\alpha_{d n}^{2}-\frac{\varepsilon_{d}}{\varepsilon_{c}} \alpha_{c n}^{2}\right)-\eta_{m}^{2}\left(1-\frac{\varepsilon_{d}}{\varepsilon_{c}}\right) \\
& Q_{7}=a^{2}\left(s^{2}+\frac{\varepsilon_{d}}{\varepsilon_{c}} k^{2}\right)-\eta_{m}^{2}\left(1-\frac{\varepsilon_{d}}{\varepsilon_{c}}\right) .
\end{aligned}
$$

\section{III. "REDUCED WAKEFIELD" IN BILAYER AREA}

Further we will assume that the channel and the area $z<0$ are vacuum $\left(n_{1}=n_{c}=1\right)$. The main attention will be given to the investigation of the sum of the wakefield (i.e., Cherenkov radiation in bilayer infinite regular waveguide) and the discrete part of the free field. This sum can be named as "reduced wakefield" (the meaning of this term will be clarified further). Hence, the reduced wakefield longitudinal component can be written as

$$
E_{2 z}^{(w)}=E_{2 z}^{(q w)}+E_{2 z}^{(b w)}
$$

Here superscripts $(q w)$ and $(b w)$ denote the wakefield and discrete part of the free field correspondingly.

Cherenkov radiation in a bilayer regular waveguide (usually named as wakefield) is determined by the poles contribution in expression (3). The wakefield explicit form can be obtained, therefore, by using the residue theorem [9]. Here we give only the expression for the wakefield longitudinal component:

$$
\begin{aligned}
E_{2 z}^{(q w)}=\sum_{k=1}^{\infty} E_{2 z k}^{(q w)}= & \frac{4 q}{v^{2}} \Theta(t) \Theta(v t-z) \operatorname{Re}\left[\sum_{k=1}^{\infty} \omega_{k}\right. \\
& \left.\times R\left(r, \omega_{k}\right) \exp \left(i \omega_{k} \frac{\zeta}{v}\right)\right]
\end{aligned}
$$


where $\Theta(x)=1$ for $x>0, \Theta(x)=0$ for $x<0$,

$$
\begin{aligned}
R(r, \omega)= & \left(\beta^{2}-1\right)\left[I_{0}(k r) \operatorname{Res}\left(\chi^{(q)}\right)\right] r \leq b, \\
R(r, \omega)= & \frac{i \pi}{2 \varepsilon_{d}}\left(n_{d}^{2} \beta^{2}-1\right)\left[\operatorname { R e s } ( \nu ^ { ( q ) } ) \left(H_{0}^{(1)}(s r)\right.\right. \\
& \left.\left.-\frac{H_{0}^{(1)}(s a)}{J_{0}(s a)} J_{0}(s r)\right)\right] r \geq b, \\
\operatorname{Res}\left(\chi^{(q)}\right)= & \frac{s K_{1}(k b) \psi_{1}(s)+\varepsilon_{d} k K_{0}(k b) \psi_{0}(s)}{d F_{c h} / d \omega}, \\
\operatorname{Res}\left(\nu^{(q)}\right)= & -\frac{2 \varepsilon_{d} k}{\pi b s} \frac{J_{0}(s a)}{d F_{c h} / d \omega} .
\end{aligned}
$$

$\operatorname{Res}[f(x)]$ means the residue of function $f(x)$, and $\omega_{k}$ is the Cherenkov frequency which is determined by the equation

$$
F_{c h}(\omega)=0,
$$

where $F_{c h}$ is defined by (17).

In accordance with (6), the longitudinal free field mode has the form

$$
\begin{aligned}
E_{2 z n}^{(b)}= & \frac{i c}{\varepsilon_{2}} \int_{-\infty}^{+\infty} \frac{B_{n}^{(2)}(\omega)}{\omega}\left(\frac{F_{n}(r, \omega)}{r}+\frac{\partial F_{n}(r, \omega)}{\partial r}\right) \\
& \times \exp \left[i \Phi_{2 n}(\omega)\right] d \omega .
\end{aligned}
$$

Asymptotic expressions for such an integral can be found by using the steepest descent method [17]. Using this method, we establish the saddle point $\omega_{n}^{(s)}$ location out of the equation $d \Phi_{2 n} / d \omega=0$, i.e., $d h_{n}^{(2)} / d \omega \cdot z-t=0$. This equation can be written in the form

$$
z=v_{n}^{(g r)}\left(\omega_{n}^{(s)}\right) t
$$

where $v_{n}^{(g r)}=\partial \omega / \partial h_{n}^{(2)}$ denotes the group velocity of the $n$ mode. Note that in the case under consideration the condition to be able to use the method of steepest descent is $\left|z-v_{n}^{(g r)}(\omega) t\right| \gg a$.

Significant contributions into the free field can be produced by the singularities of the integrand (27), which are crossed at transformation of the initial integration path (real axis) toward the steepest descending path (SDP). The desired discrete part of the free field is determined by those of the crossed singularities, which represent the poles of function $B_{n}^{(2)}(\omega)$.

In the case $n_{1}=n_{c}=1$ the right part $\vec{U}$ of the system (13) is simplified:

$$
\begin{aligned}
U_{m}= & \frac{q \omega a k}{\pi v c\left(k^{2} a^{2}+\eta_{m}^{2}\right)\left(s^{2} a^{2}-\eta_{m}^{2}\right)} \\
& \times \frac{s a J_{1}\left(\eta_{m} b / a\right) \psi_{1}(s) Q_{3}-\eta_{m} J_{0}\left(\eta_{m} b / a\right) \psi_{0}(s) Q_{4}}{F_{c h}(\omega)} .
\end{aligned}
$$

Comparing the function $U_{m}(\omega)$ denominator with the equation for Cherenkov frequencies (26), we conclude that the poles of the function $U_{m}(\omega)$ are at frequencies $\pm \omega_{k}(k=1,2 \ldots)$. The functions $B_{n}^{(2)}(\omega)$ may have poles only at the same frequencies as the function $U_{m}(\omega)$.

After cumbersome mathematical transformations one can show that

$$
\begin{aligned}
\operatorname{Res}\left(B_{n}^{(2)}\left(\omega_{k}\right)\right)= & -\frac{i q \varepsilon_{d} k\left(\omega_{k}\right)}{\pi c b} \frac{\psi_{0}\left[s\left(\omega_{k}\right)\right]}{I_{1}\left[k\left(\omega_{k}\right) b\right]} \\
& \times\left.\left[\frac{d F_{c h}}{d \omega}\right]^{-1}\right|_{\omega=\omega_{k}} \text { if } k=n, \\
\operatorname{Res}\left[B_{n}^{(2)}\left(\omega_{k}\right)\right]= & 0 \quad \text { if } k \neq n,
\end{aligned}
$$

i.e., the amplitude of the $n$th free field mode possesses only one pole from the complete set of Cherenkov frequencies. The contribution of the pole $\omega_{k}$ is determined by the residue theorem. Here we present only the longitudinal component of the free field discrete part:

$$
\begin{aligned}
E_{2 z}^{(b w)}= & \frac{4 \pi c}{\varepsilon_{2}} \Theta(t) \operatorname{Re}\left[\sum_{k=1}^{\infty} \frac{\operatorname{Res}\left[B_{k}^{(2)}\left(\omega_{k}\right)\right]}{\omega_{k}}\right. \\
& \times\left(\frac{F_{k}\left(r, \omega_{k}\right)}{r}+\frac{\partial F_{k}\left(r, \omega_{k}\right)}{\partial r}\right) \\
& \left.\times \Theta\left[v_{k}^{(g r)}\left(\omega_{k}\right) t-z\right] e^{i \frac{\zeta}{2} \omega_{k}}\right] .
\end{aligned}
$$

As can be seen, the discrete part of the free field consists of a set of waves (Cherenkov modes) generated at Cherenkov frequencies.

The contribution (31) exists only if the pole $\omega_{k}$ is crossed at transformation of the initial integration path (real axis) toward the SDP. One can show that this crossing takes place under condition $\omega_{k}^{(s)}<\omega_{k}$, that is identical with inequality $z<v_{k}^{(g r)}\left(\omega_{k}\right) t$. Further, one can show that the pole contribution is equal in magnitude and has the opposite sign in comparison with wakefield mode (25) generated at the same frequency $\omega_{k}$. Thus, the wakefield mode is compensated by the corresponding part of the free field in the area $z<v_{k}^{(g r)}\left(\omega_{k}\right) t$. Therefore, the mode of the reduced wakefield in the area $z<v_{k}^{(g r)}\left(\omega_{k}\right) t$ is equal to zero. This effect determines the choice of the term reduced wakefield used to denote the sum of the wakefield and discrete part of the free field. As soon as the group velocity $v_{k}^{(g r)}\left(\omega_{k}\right)$ is different for different modes and, moreover, increases with increase of mode number, we observe the effect of switching off the modes one by one behind the charge. The analogous effect was described in Ref. [12] for the case of homogeneous dielectric filling in both waveguide areas.

The obtained results can be generalized for the case of a bunch having Gaussian longitudinal profile with volume 
charge density $\rho^{(g)}=q(\sqrt{2 \pi} \sigma)^{-1} \delta(x) \delta(y) \exp \left[-\zeta^{2} /\left(2 \sigma^{2}\right)\right]$. As a result, the longitudinal component of the reduced wakefield generated by Gaussian bunch in a bilayer waveguide area can be written in the form

$$
\begin{aligned}
E_{2 z}^{(w)}= & \frac{4 q}{v^{2}} \Theta(t) \Theta(-\zeta) \operatorname{Re}\left[\sum_{k=1}^{\infty} \omega_{k} R\left(r, \omega_{k}\right) \Theta\left[z-v_{k}^{(g r)}\left(\omega_{k}\right) t\right]\right. \\
& \left.\times \exp \left(i \frac{\zeta}{v} \omega_{k}-\frac{\left(\omega_{k} \sigma\right)^{2}}{2 v^{2}}\right)\right] .
\end{aligned}
$$

As follows from this expression, it is possible to achieve an amplitude decrease of the high-frequency modes by bunch length selection, since there is a summand $\exp \left[-\left(\omega_{k} \sigma\right)^{2} /\left(2 v^{2}\right)\right]$.

\section{NUMERICAL RESULTS AND DISCUSSION}

Figure 2 shows the example of the time dependence of the reduced wakefield component $E_{2 z}^{(w)}$ at point $r=0 \mathrm{~cm}$, $z=75 \mathrm{~cm}$ in the case in moving point charge (waveguide and particle parameters are $a=1 \mathrm{~cm}, b=0.2 \mathrm{~cm}, \varepsilon_{d}=4$, $\left.\mu_{d}=1, q=1 \mathrm{nC}, v=0.99 c\right)$. We present the case when the wakefield and the discrete part of free field practically contain 16 Cherenkov modes (further increase in number of modes leads to insignificant change of the wave field). As was mentioned earlier, the $k$ th mode of the reduced wakefield exists in the area $z>v_{k}^{(g r)}\left(\omega_{k}\right) t$ only. We emphasize that the end of this area moves with the group velocity of the mode. The reduced wakefield $E_{2 z}^{(w)}$ is consequently composed of a different number of modes depending on the time moment and the observation point. The number $i$ in Fig. 2 means the number of modes in the corresponding time interval. One can see that the number of modes in the field $E_{2 z}^{(w)}$ decreases with increasing distance from the charge. In time domain $2.53<t<8.17 \mathrm{~ns}$ the reduced wakefield contains the set of sharp extrema. A similar field structure corresponds, as a rule, to the field in a wakefield accelerator [6]. From the moment $t \approx 9.84 \mathrm{~ns}$ we can see a single frequency field. The reduced wakefield disappears at moment $t \approx 9.90 \mathrm{~ns}$.

Figure 3 shows the dependence of the relative group velocity $v_{k}^{(g r)}\left(\omega_{k}\right) / c$ on the mode number $k$ for the same structure parameters. As one can see, the group velocity of the first mode is close to the group velocity of the first mode in the case of homogeneous dielectric waveguide, i.e., $v_{1}^{(g r)}\left(\omega_{1}\right) \approx c^{2} /\left(v \varepsilon_{d}\right) \approx c / \varepsilon_{d}=0.25 c$ This effect can be
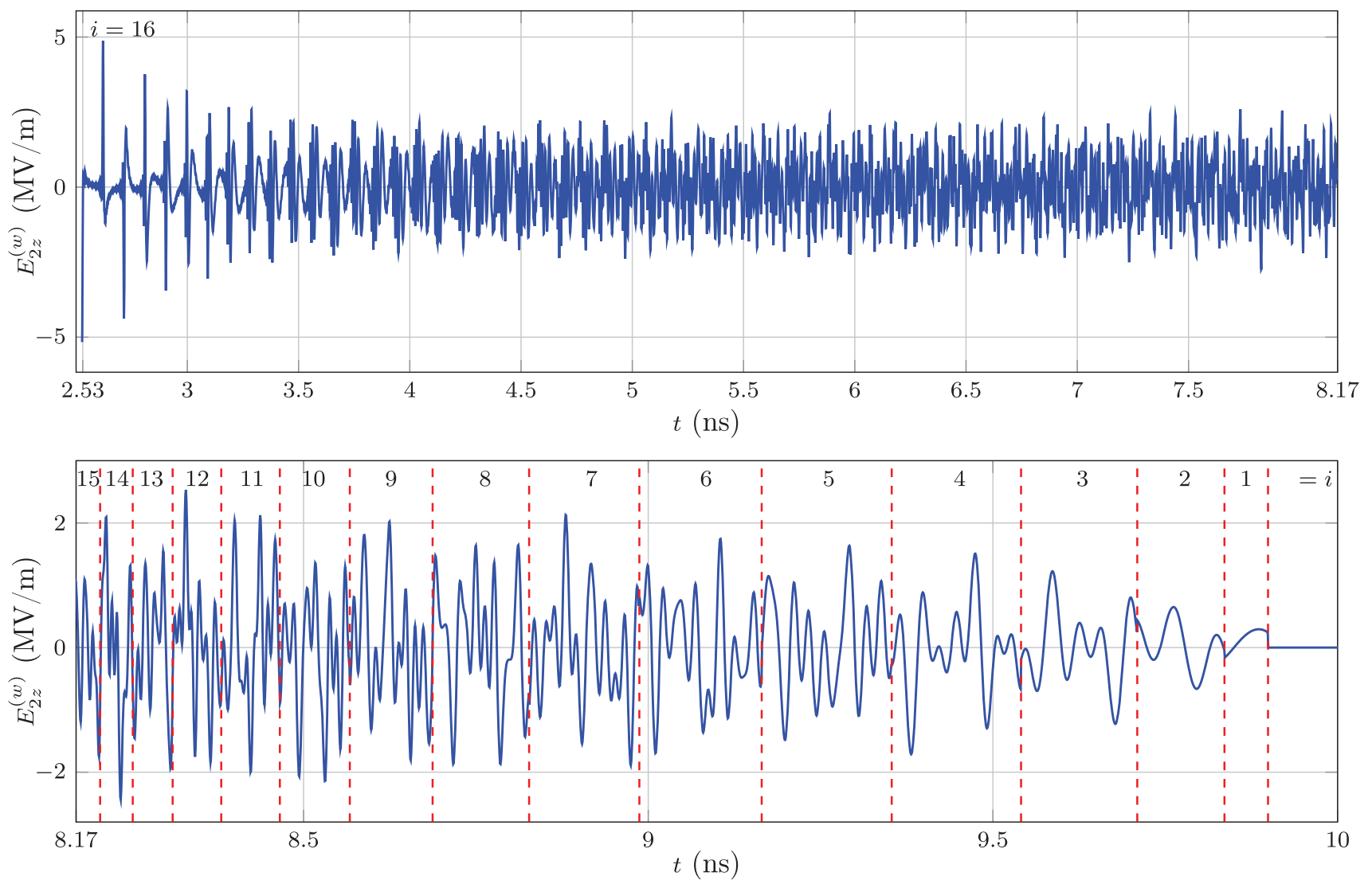

FIG. 2. Dependence of the reduced wakefield longitudinal component $E_{2 z}^{(w)}$ on time $t$ at point $r=0 \mathrm{~cm}, z=75 \mathrm{~cm}$ (obtained on the basis of analytical results). Waveguide and point charge parameters are $a=1 \mathrm{~cm}, b=0.2 \mathrm{~cm}, \varepsilon_{d}=4, \mu_{d}=1, q=1 \mathrm{nC}, v=0.99 c$. The number $i$ means the number of Cherenkov modes contributing in a corresponding time interval. 


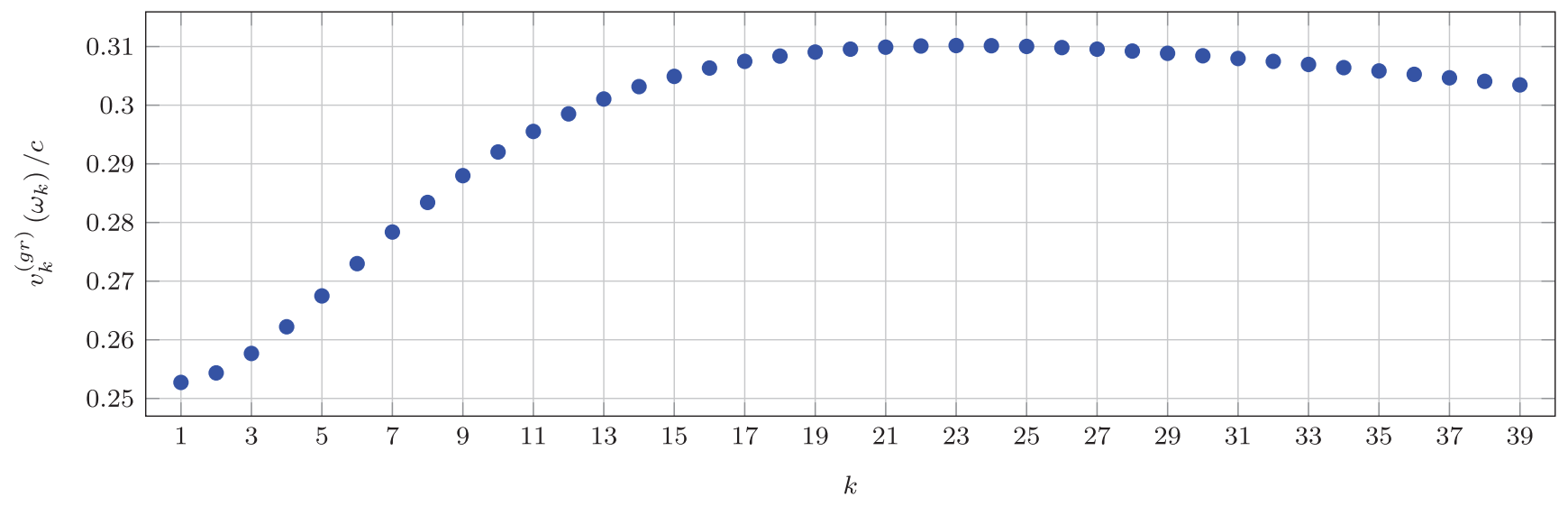

FIG. 3. Dependence of the relative group velocity $v_{k}^{(c h)}\left(\omega_{k}\right) / c$ on mode number $k$ at point $r=0 \mathrm{~cm}, z=75 \mathrm{~cm}$. Waveguide and point charge parameters are the same as in Fig. 2.

explained by the fact that characteristic scales of the first modes variation are large in comparison with the channel radius, so the channel has only a little influence on these modes. Note that the group velocity behavior becomes more complicated with increasing of the mode number. For small mode numbers the group velocity slowly increases with $k$, then faster and reaches a maximum (its further decrease is not essential since the modes with number greater than 16 are insignificant for the parameters under consideration). Such behavior leads to the unequal time intervals in Fig. 2.

The results based on the analytical investigation are verified by comparing with results of numerical simulation in CST Particle Studio for the case of a moving Gaussian bunch. Figure 4 illustrates the time dependence of the total field $E_{2 z}^{(\mathrm{CST})}$ calculated by CST simulation at point $r=0.3 \mathrm{~cm}, z=30 \mathrm{~cm}$ and the same dependence $E_{2 z}^{(w)}$ for a reduced wakefield obtained on the basis of analytical consideration. The following model is considered: $a=1 \mathrm{~cm}, b=0.7 \mathrm{~cm}, \varepsilon_{d}=4, \mu_{d}=1, q=1 \mathrm{nC}, \sigma=0.6 \mathrm{~cm}$, $v=0.9 c$. The bilayer waveguide part is $100 \mathrm{~cm}$ in length and the vacuum part is $30 \mathrm{~cm}$ in the CST model. The parameter $\sigma$ was chosen so that the wakefield excited in this situation has practically only the first mode with frequency $\omega_{1} \approx 13.0 \mathrm{GHz}$ (other modes have negligible amplitudes). The moment $t_{1} \approx 2.84 \mathrm{~ns}$ corresponds to the time when condition $z=v_{1}^{(g r)}\left(\omega_{1}\right) t$ is satisfied at the observation point ("breakage" of the wakefield mode). One can see that the total field coincides with the wakefield at $t<t_{1}$ almost everywhere with very high accuracy (in the domain $t<t_{1}$ the pole contribution of the free field is absent). The wave parts of the forced and the free fields compensate each other for $t>t_{1}$; therefore the reduced wakefield is absent in this domain. Naturally, the exact field (blue curve) is continuous: there is some transient process in the total field at $t \sim t_{1}$. Note that the amplitude of the exact field is approximately equal to half of the amplitude of forming the wakefield at $t=t_{1}$. Mathematically this point corresponds

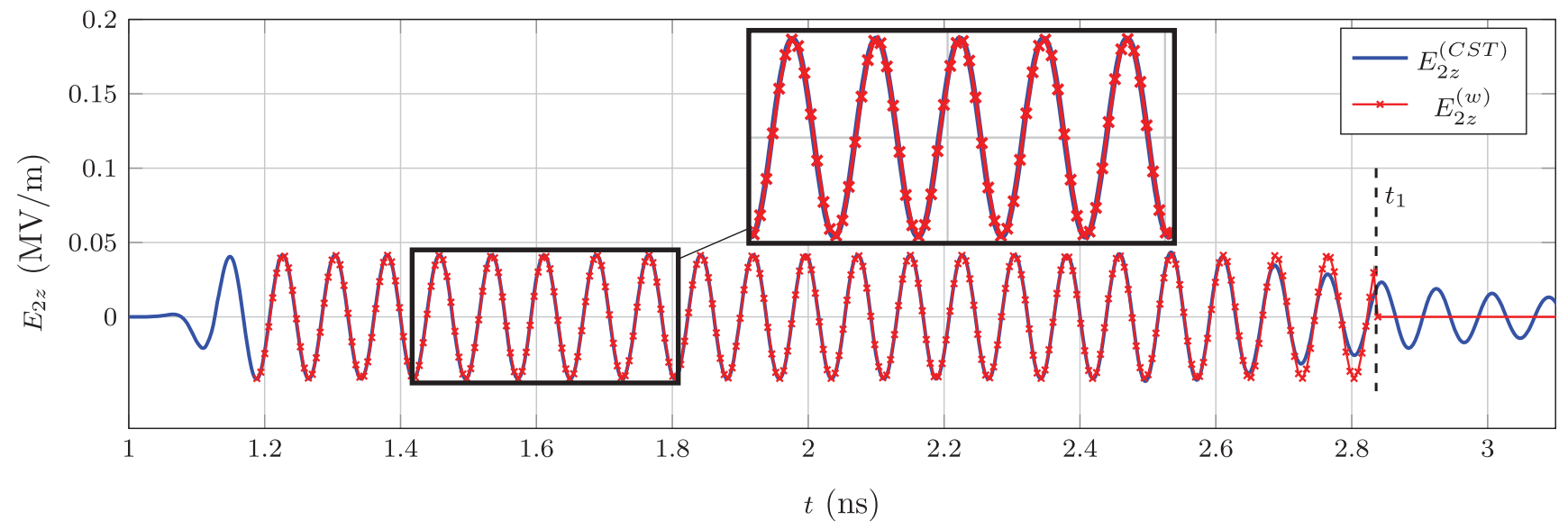

FIG. 4. Dependence of component $E_{2 z}^{(\mathrm{CST})}$ of the total field obtained using CST simulation (blue solid line) and reduced wakefield component $E_{2 z}^{(w)}$ obtained from analytical investigation (red marked line) on time $t$ at point $r=0.3 \mathrm{~cm}, z=30 \mathrm{~cm}$. Waveguide and bunch parameters are $a=1 \mathrm{~cm}, b=0.7 \mathrm{~cm}, \varepsilon_{d}=4, \mu_{d}=1, q=1 \mathrm{nC}, \sigma=0.6 \mathrm{~cm}, v=0.9 c$. 
to coincidence of the pole and the saddle point where the integral asymptotic is determined by half of the pole contribution [17].

\section{CONCLUSION}

We have investigated the electromagnetic radiation generated by the charge crossing the boundary between the vacuum area of the cylindrical waveguide and the area with the layered dielectric filling. The solution was carried out by representing the free field as a set of eigenmodes in corresponding waveguide areas. Primary attention was given to the study of the wave field in the region partially loaded with a dielectric. It was shown that wakefield and free field discrete parts are excited at the same Cherenkov frequencies and compensate each other in some area behind the charge. Results obtained for the point source were generalized to the bunch with a Gaussian longitudinal profile.

The typical wakefield formation process in a bilayer waveguide area was presented. At the given observation point, the reduced wakefield is simplified with time (the number of modes is decreased). An analogous effect takes place at the given observation moment: the number of modes decreases with increasing distance from the charge to the observation point. To verify the analytical results, we used the numerical simulation by CST for the case of Gaussian bunch. Simulations show that there is the large domain behind the bunch where the total electromagnetic field is almost entirely determined by the wakefield only. As well, there is some transition domain where the mode amplitude is smoothly decreased.

\section{ACKNOWLEDGMENTS}

This work is supported by Russian Foundation for Basic Research according to the research Project No. 1502-03913.

[1] W. Gai, P. Schoessow, B. Cole, R. Konecny, J. Norem, J. Rosenzweig, and J. Simpson, Experimental Demonstration of Wake-Field Effects in Dielectric Structures, Phys. Rev. Lett. 61, 2756 (1988).

[2] A. Kanareykin, Cherenkov radiation and dielectric based accelerating structures: wakefield generation, power extraction and energy transfer efficiency, J. Phys. Conf. Ser. 236, 012032 (2010).

[3] L. Xiao, W. Gai, and X. Sun, Field analysis of a dielectricloaded rectangular waveguide accelerating structure,
Proceedings of the Particle Accelerator Conference, 2001 (IEEE, New York, 2001), pp. 3963-3965.

[4] S. S. Baturin, I. L. Sheinman, A. M. Altmark, D. A. Semikin, and A. D. Kanareykin, Wakefield radiation generated in a dielectric-filled rectangular accelerating structure, Tech. Phys. Lett. 37, 394 (2011).

[5] S. S. Baturin, I. L. Sheinman, A. M. Altmark, and A. D. Kanareikin, Wakefield radiation generated by an electron bunch in a rectangular dielectric waveguide, Tech. Phys. 57, 683 (2012).

[6] M. Rosing and W. Gai, Longitudinal- and transverse-wakefield effects in dielectric structures, Phys. Rev. D 42, 1829 (1990).

[7] B. D. O'Shea, G. Andonian, S. K. Barber, K. L. Fitzmorris, S. Hakimi, J. Harrison, P. D. Hoang, M. J. Hogan, B. Naranjo, O. B. Williams, V. Yakimenko, and J.B. Rosenzweig, Observation of acceleration and deceleration in gigaelectron-volt-per-metre gradient dielectric wakefield accelerators, Nat. Commun. 7, 12763 (2016).

[8] W. Li, Z. He, and Q. Jia, Electron bunch length measurement with a wakefield radiation decelerator, Phys. Rev. ST Accel. Beams 17, 042801 (2014).

[9] A. V. Tyukhtin, Determination of the particle energy in a waveguide with a thin dielectric layer, Phys. Rev. ST Accel. Beams 15, 102801 (2012).

[10] T. Yu. Alekhina and A. V. Tyukhtin, Electromagnetic field of a charge intersecting a cold plasma boundary in a waveguide, Phys. Rev. E 83, 066401 (2011).

[11] T. Yu. Alekhina, A. V. Tyukhtin, and A. A. Grigoreva, Cherenkov-transition radiation in a waveguide partly filled with a resonance dispersion medium, Phys. Rev. ST Accel. Beams 18, 091302 (2015).

[12] T. Yu. Alekhina and A. V. Tyukhtin, Radiation of a charge in a waveguide with a boundary between two dielectrics, J. Phys. Conf. Ser. 357, 012010 (2012).

[13] A. A. Grigoreva, A. V. Tyukhtin, V. V. Vorobev, T. Yu. Alekhina, and S. Antipov, Mode transformation in a circular waveguide with a transverse boundary between a vacuum and a partially dielectric area, IEEE Trans. Microwave Theory Tech. 64, 3441 (2016).

[14] A. A. Grigoreva, A. V. Tyukhtin, V. V. Vorobev, and S. Antipov, Radiation of a charge intersecting a boundary between a bilayer area and a homogeneous one in a circular waveguide, IEEE Trans. Microwave Theory Tech. 66, 49 (2018).

[15] V. L. Ginzburg and V. N. Tsytovich, Transition Radiation and Transition Scattering (Adam Hilger, London, 1990).

[16] B. M. Bolotovskii, Theory of cherenkov radiation (iii), Phys. Usp. 4, 781 (1962).

[17] L. B. Felsen and N. Marcuvitz, Radiation and Scattering of Waves (Prentice-Hall, New York, 1972). 